Original Research Paper

\title{
Thermoelectric and Optical Properties of Advanced Thermoelectric Devices from Different Multilayer Thin Films
}

\author{
${ }^{1}$ Mebougna Drabo and ${ }^{2}$ Satilmis Budak \\ ${ }^{1}$ Department of Mechanical and Civil Engineering, Alabama A\&M University, Normal, AL USA \\ ${ }^{2}$ Department of Electrical Engineering and Computer Science, Alabama A\&M University, Normal, AL USA
}

\section{Article history}

Received: 17-05-2019

Revised: 21-06-2019

Accepted: 27-08-2019

Corresponding Author:

Satilmis Budak

Department of Electrical

Engineering and Computer

Science,

Alabama A andM University,

Normal, AL USA

Email: satilmis.budak@aamu.edu

\begin{abstract}
Thermoelectric devices were prepared using different multilayered thin film structures in the order of $\mathrm{Ge} / \mathrm{Si}+\mathrm{Ge}, \mathrm{Si}+\mathrm{Ge}, \mathrm{Si}$, $\mathrm{Sb}+\mathrm{Ge}, \mathrm{Ge}$ and $\mathrm{SiO}_{2} / \mathrm{SiO}_{2}+\mathrm{Ge}$ by DC/RF Magnetron Sputtering. The thickness of the deposited thin films were measured using Filmetrics UV thickness measurement system. The prepared thermoelectric devices have been modified with $5 \mathrm{MeV} \mathrm{Si}$ ions bombardments at the different fluences (doses) using Pelletron high energy beam accelerator at Alabama A and M University to form quantum structures in the multilayer thin films to improve the efficiency of the thermoelectric devices. The conductivity measurements, the fluence dependence of the power factor measurements, the fluence dependence of thermal conductivity measurements and the figure of merit calculations of multilayered thin films at room temperature have been performed. After the thermoelectric devices were prepared, Xray Diffraction, Scanning Electron Microscopy (SEM) + Energy-Dispersive X-beam Spectroscopy (EDS) and Scanning Transmission Electron Microscopy (TEM) measurements have been done from the cross section of the prepared multilayer thin films to give us some answer for the thickness of the fabricated thermoelectric devices.
\end{abstract}

Keywords: Thermoelectric Materials, Figure of Merit, Seebeck Coefficient, High Energy Ion Beam Bombardment

\section{Introduction}

\section{Thermoelectric Materials}

Thermoelectric materials are considered to be any substance that can convert heat energy to electrical energy supported by evidence found by Seebeck and Peltier (Zhang and Zhao, 2015). The materials have an element placed between a heat source and the ambient (heat sink). This transfer of heat from the source to the sink is achieved either through the carriers' motion (via electrons/holes) or through the lattice (via collective lattice vibration modes/phonons). The heat/carrier transport results in a potential difference-which is termed the Seebeck voltage $(\Delta \mathrm{V})$ (Pichanusakorn and Bandaru, 2010). The thermopower/Seebeck coefficient $\mathrm{S}$ is then the ratio of $\Delta \mathrm{V}$ to the temperature difference $(\Delta \mathrm{T})$ (Fig. 1). The Peltier coefficient (II), also called the proportionality constant, represents that the carrier transport flow through a conductor carries heat: Q $=$ III where $\mathrm{Q}$ is the heat current (proportional to I) and I is the charge current (Chen et al., 2003).
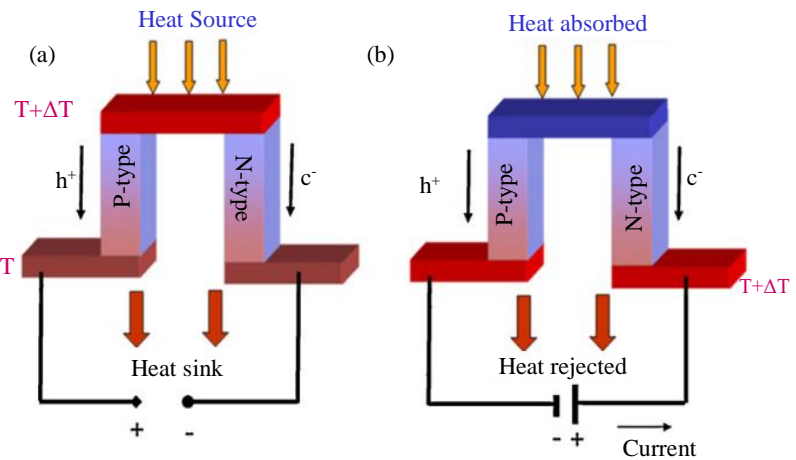

Fig. 1: Basic thermoelectric device for (a) heat-to-electrical energy conversion and (b) heat pumping (Pichanusakorn and Bandaru, 2010)

Thermoelectric materials generally have three parameters that simultaneously depend on carrier concentration: Large Seebeck coefficient, high electrical conductivity and low thermal conductivity. Recent studies have shown that these requirements are difficult to satisfy (Zhang and Zhao, 2015). This may lead to 
significantly reduced or almost lost thermal energy that could have be applied elsewhere more efficiently. The efficiency of Conversion of Performance (COP) is shown using the following equation: $Z T=\mathrm{S}^{2} \sigma \mathrm{T} / \mathrm{K}$ where $Z T$ is the figure of merit, $S$ is the Seebeck coefficient, $\mathrm{K}$ is the thermal conductivity, $\sigma$ is the electrical conductivity and $T$ is absolute temperature. Those materials with low thermal conductivity and high electrical conductivity are mandated to acquire high $Z T$ or alternatively, higher efficiency of the material (Pichanusakorn and Bandaru, 2010).

\section{Nanostructured Thermoelectric Materials}

What researchers have found so far is the potential for compound semiconductor quantum wells, superlattice structures, $S i$ nanowires, quantum dot and other nanostructure incorporated thermoelectrics to have a higher ZT and energy conversion efficiency (Pichanusakorn and Bandaru, 2010). If these could be optimized and implemented, then there would be a high demand of practical utilization to replace outdated systems with solid state energy structures (Chen et al., 2003). It has been concluded in recent studies that the majority of the apparent increase in the figure of merit of nanostructured thermoelectrics has been accomplished by means of an extreme reduction of the lattice thermal conductivity $\left(\mathrm{K}_{\mathrm{L}}\right)$. This reduction has provided a huge motivation for the use of nanostructures and will continue to be researched in future studies (Pichanusakorn and Bandaru, 2010).

We have reached some remarkable results on the $\mathrm{SiO}_{2} / \mathrm{SiO}_{2}+\mathrm{Ge}, \mathrm{Si} / \mathrm{Si}+\mathrm{Ge}$ and $\mathrm{Si} / \mathrm{Si}+\mathrm{Sb}$ multilayer thin film systems under the high energy ion beam modification. At the suitable fluence (doses) of the high energy $\mathrm{Si}$ ions could cause increase in Seebeck coefficient and the electrical conductivity, while decrease in the thermal conductivity (Budak et al., 2013; 2014 ; 2015). We would like to combine some previous multilayer structures under one form of the thermoelectric device to see the effect of the high energy ion beam effects on the thermoelectric properties for the energy harvesting thermoelectric devices. In the previous study (Budak et al., 2016), some of the thermoelectric and optical properties were published including, Seebeck coefficient, resistivity, density, Hall coefficient, mobility, some of FIB and SEM/EDS results and SRIM simulation. In the current study, addition to the uses of Seebeck and resistivity data from the previous work (Budak et al., 2016), some additional measurements and calculations were established to give more details about the thermoelectric thin film systems for high efficient thermoelectric applications.

\section{Experimental Program}

Figure 2 shows the geometry during the deposition of the different multilayer thin films from $\mathrm{Ge} / \mathrm{Si}+\mathrm{Ge}$, $\mathrm{Si}+\mathrm{Ge}, \mathrm{Si}, \mathrm{Sb}+\mathrm{Ge}, \mathrm{Ge}$ and $\mathrm{SiO}_{2} / \mathrm{SiO}_{2}+\mathrm{Ge}$ materials from the top of the thin films to the bottom of the thin films. After $\mathrm{SiO}_{2}$ substrates were cut into small pieces using the diamond cutter, they were loaded on the Aluminum substrate holder using double side sticky carbon tape. The Aluminum substrate holder was loaded into the vacuum chamber of the DC/RF Magnetron Sputtering system. The chamber was started to be evacuated using mechanical pump and the turbo molecular pump. When the vacuum inside the chamber reached about $3 \times 10^{-5}$ Torr, the Ar gas was introduced to start deposition of the multilayer thin films. When the Ar gas was introduced, the background vacuum of about $3 \times 10^{-5}$ Torr dropped to about $3 \times 10^{-3}$ Torr. This vacuum is good enough to start the deposition of the multilayer thin films using DC and RF sources under the Ar gas. First, 6 alternating layers of the $\mathrm{SiO}_{2}$ and $\mathrm{SiO}_{2}+\mathrm{Ge}$ thin films were deposited, then single layer of $\mathrm{Ge}$ thin film was deposited. The $\mathrm{SiO}_{2}$ target was replaced with $\mathrm{Sb}$ target and single layer of $\mathrm{Sb}+\mathrm{Ge}$ thin film was deposited. Sb target was replaced with Si and single layer of Si thin film deposited. Then, single layer of $\mathrm{Si}+\mathrm{Ge}$ thin film deposited at the top of the previous multilayers. Finally, 100 alternating layers of $\mathrm{Ge}$ and $\mathrm{Si}+\mathrm{Ge}$ thin films were deposited at the top of the whole thin films. The thickness of the deposited thin films were measured using Filmetrics UV thickness measurement system as seen from Fig. 2.

\begin{tabular}{|c|c|}
\hline 100 layers & $\mathrm{Ge} / \mathrm{Si}+\mathrm{Ge}$ \\
\hline Single layers & $\mathrm{Si}+\mathrm{Ge}$ \\
\hline Single layers & $\mathrm{Si}$ \\
\hline Single layers & $\mathrm{Sb}+\mathrm{Ge}$ \\
\hline Single layers & $\mathrm{Ge}$ \\
\hline \multirow[t]{2}{*}{6 layers } & $\mathrm{SiO}_{2} / \mathrm{SiO}_{2}+\mathrm{Ge}$ \\
\hline & Substrate \\
\hline
\end{tabular}

Fig. 2: Geometry for the preparation of the thermoelectric device 
Also, Focused Ion Beam (FIB) and Transmission Electron Microscopy (TEM) measurements have been done from the cross section of the prepared multilayer thin films to give us some answers for the thickness of the fabricated thermoelectric devices. The prepared thermoelectric devices have been modified with $5 \mathrm{MeV}$ $\mathrm{Si}$ ions bombardments at the different fluences (doses) using Pelletron high energy beam accelerator at Alabama $\mathrm{A}$ and $\mathrm{M}$ University to form quantum structures in the multilayer thin films to improve the efficiency of the thermoelectric devices.

\section{Results}

Figure 3 shows the electrical conductivity measurements of the multilayered thin films bombarded at different fluences. The four probe resistivity measurements have been performed when the van der Pauw measurement system's operation temperature was increased from $300 \mathrm{~K}$ to $500 \mathrm{~K}$. The electrical resistivity measurement have been measured for the prepared devices from the different multilayer thin films. Then, the electrical conductivity parameters were calculated by taking the reciprocal of the resistivity measurements and plotted in Fig. 3. As seen from Fig. 3, suitable does of high energy ion beam caused an increase in the electrical conductivity values. The increase in the electrical conductivity is one of the expected thing from high efficient thermoelectric materials and devices.

Many scientists use the power factor for the thermoelectric materials and devices as one type of efficiency parameter. The power factor could be calculated with the multiplication of square of the Seebeck coefficient and the electrical conductivity values. Figure 4 shows the fluence dependence of the power factor measurements of the multilayered thin films at room temperature. As seen from Fig. 4, the highest power factor could be reached when the high energy beam dose (fluence) was $1 \times 10^{13}$ ions $/ \mathrm{cm}^{2}$. It seems that the fluence of $1 \times 10^{13}$ ions $/ \mathrm{cm}^{2}$ looks like a turning point since the power factor increases until this fluence and then the power factor started decrease after this fluence. The similar behaviors could be seen in Fig. 3 for the electrical conductivity values.

Figure 5 shows the fluence dependence of thermal conductivity measurements of the multilayered thin films at room temperature. As seen from Fig. 5, the highest thermal conductivity could be reached when the high energy beam dose (fluence) was $1 \times 10^{13}$ ions $/ \mathrm{cm}^{2}$. It seems that the fluence of $1 \times 10^{13}$ ions $/ \mathrm{cm}^{2}$ looks like a turning point since the thermal conductivity increases until this fluence and then the thermal conductivity started decrease after this fluence. Efficient thermoelectric materials and devices could have high Seebeck coefficient and electrical conductivity and lower thermal conductivity values.

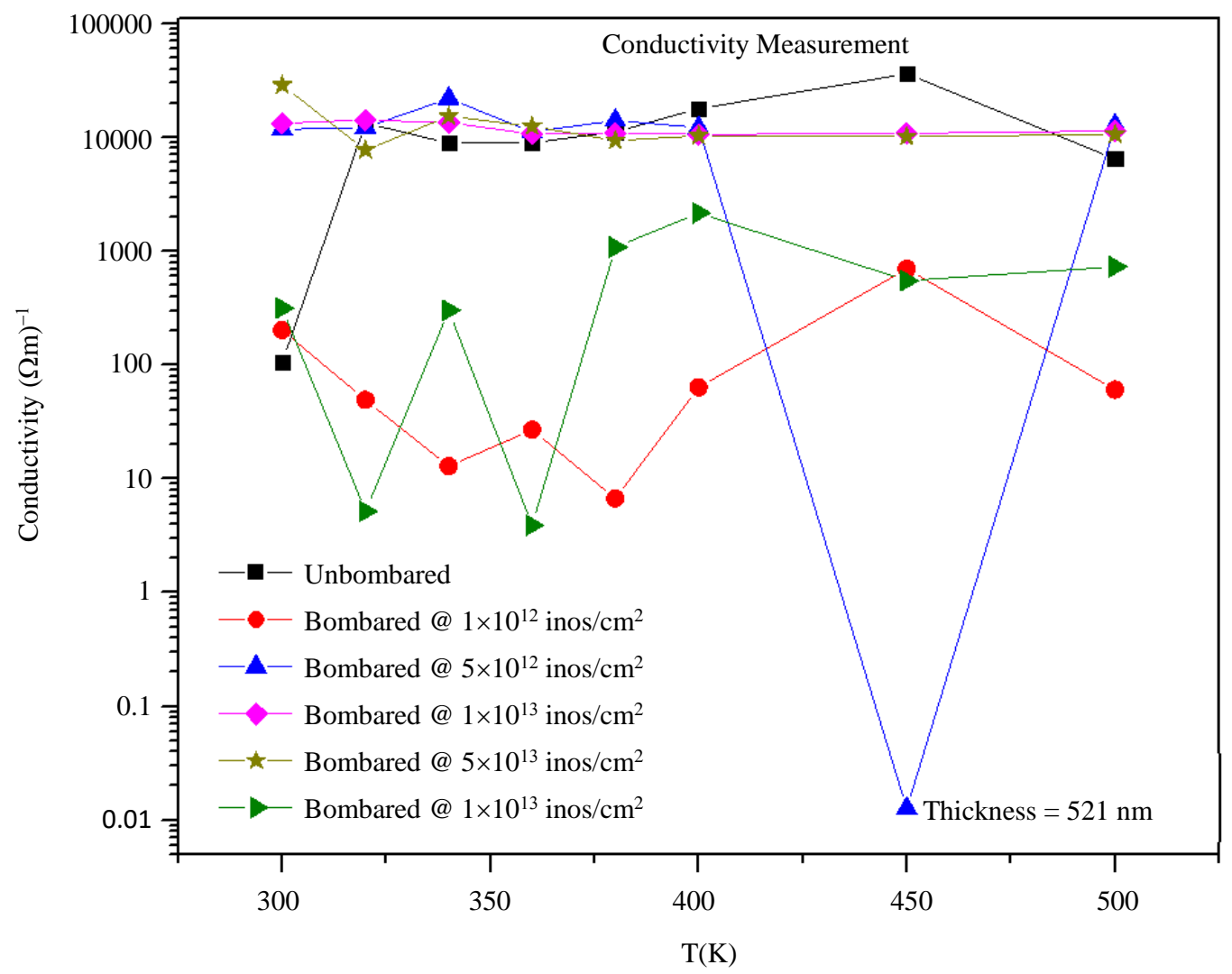

Fig. 3: Electrical conductivity measurement of the multilayered thin films 


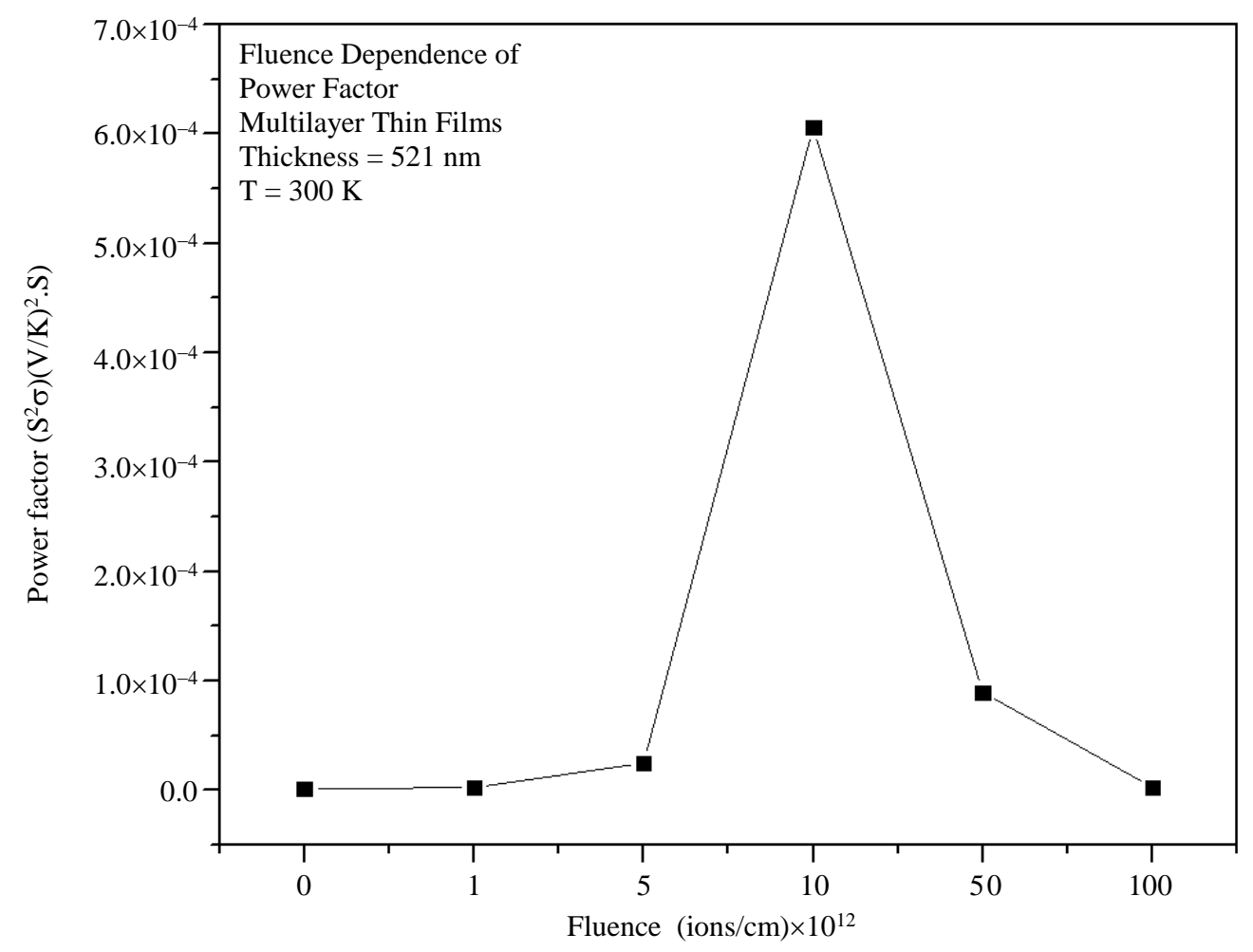

Fig. 4: Fluence dependence of the power factor of the multilayered thin films at room temperature

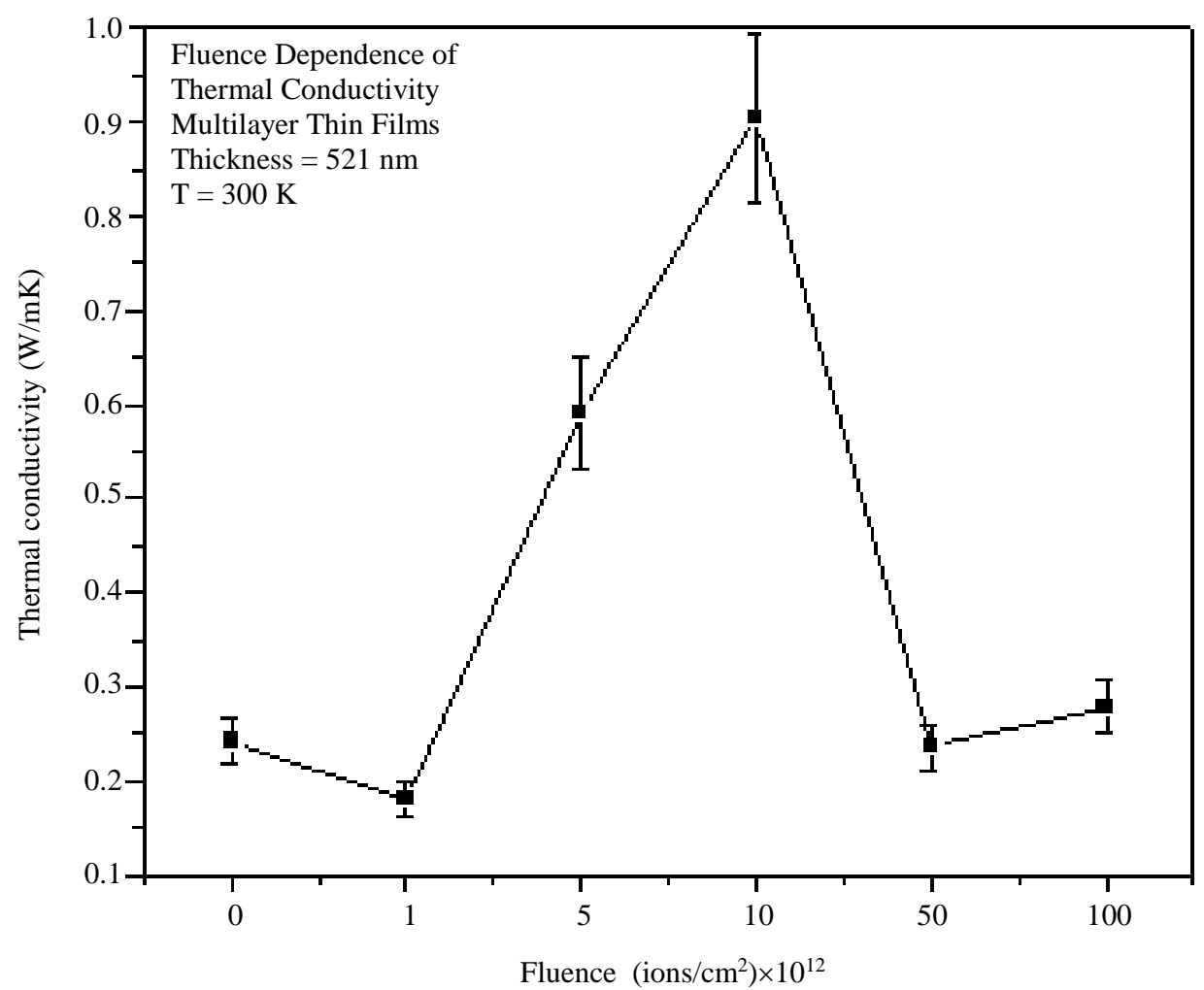

Fig. 5: Fluence dependence of the thermal conductivity measurements of the multilayered thin films at room temperature 
After the fluence of $1 \times 10^{13}$ ions $/ \mathrm{cm}^{2}$, the thermal conductivity values decrease. This a good sign for the efficient thermoelectric devices. But, the figure of merit could be calculated by the multiplication of the square of high energy ion beam bombardment with electrical conductivity and absolute temperature and dividing of all of them with the thermal conductivity. The efficient thermoelectric devices should have high figure of merit, ZT. Figure 6 shows the fluence dependence of the Figure of Merit, ZT of the multilayered thin films at room temperature. Since ZT value is more important, even the experiments show some high thermal conductivity, one could have higher figure of merit and then all of the parameters are considered including Seebeck coefficient, electrical conductivity and thermal conductivity. As seen from Fig. 6, the highest figure if merit, ZT, was reached at the fluence of $1 \times 10^{13}$ ions $/ \mathrm{cm}^{2}$ with the value of 0.20 . This fluence (dose) looks like a turning point for this material system since the the figure of merit started to decrease after the fluence of $1 \times 10^{13}$ ions $/ \mathrm{cm}^{2}$.

\section{X-Ray Diffraction}

$\mathrm{X}$-rays are electromagnetic radiation with a wavelength range between 0.01 and 100 angstroms (A) (He, 2018). X-ray diffraction occurs in the range of $1 \AA$. X-ray diffraction is defined as the scattering of X-rays by a sample's (usually crystalline) atoms based on their uniform spacing. This scattering produces a patterned diffraction interference effect which provides information on the structure of the substance. The structure can also help to identify what type of crystal it is (Fig. 7). This is because the scattered Xrays are not evenly disbursed but are a function of the electron distribution in the sample (He, 2018).

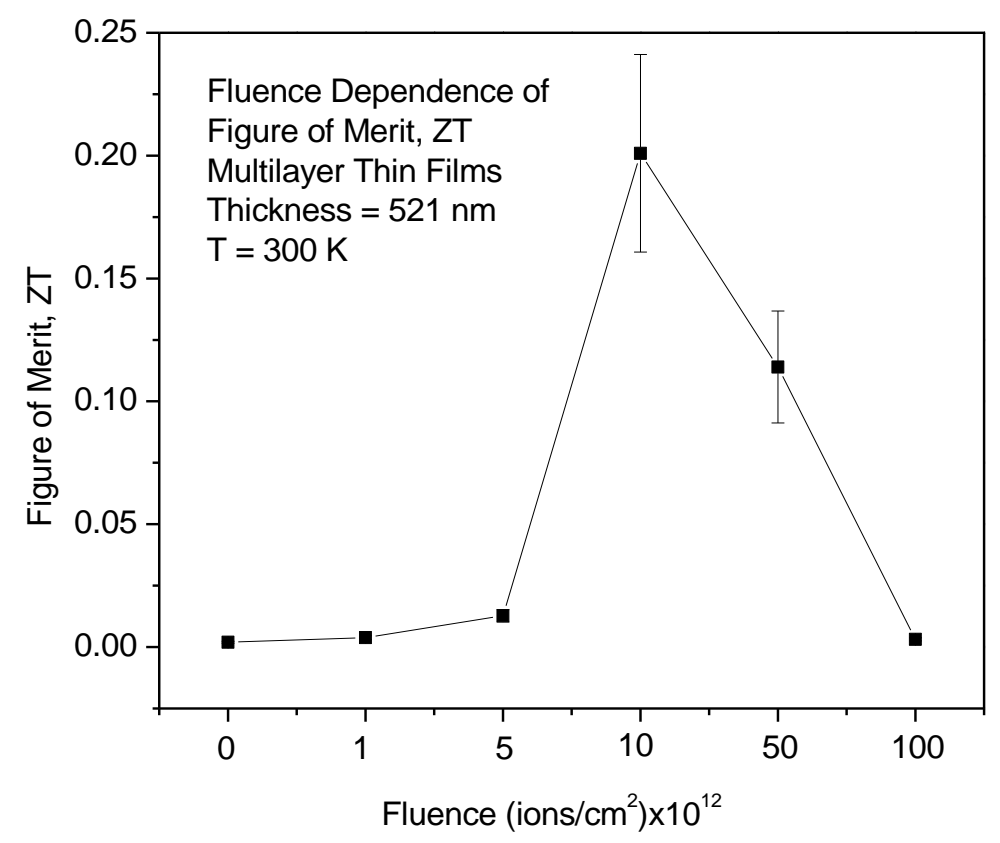

Fig. 6: Fluence dependence of the Figure of Merit, ZT of the multilayered thin films at room temperature

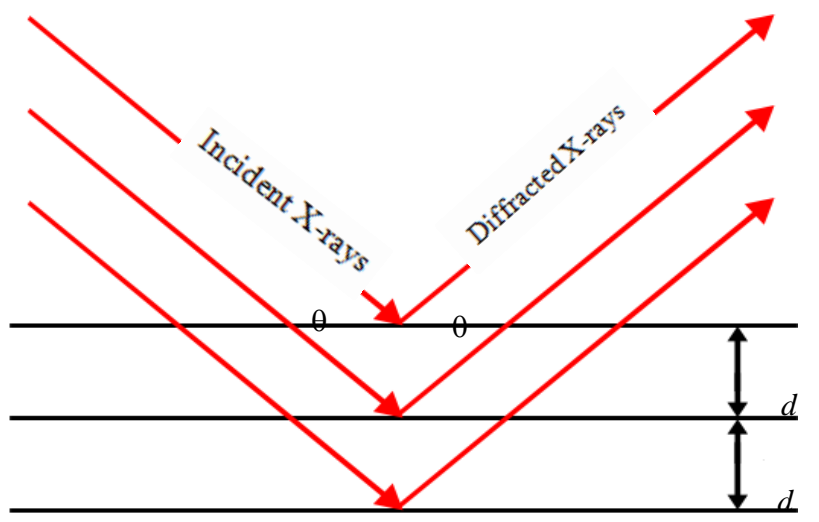

Fig. 7: X-rays make an angle of $\theta$ symmetric to the normal of crystal plane 


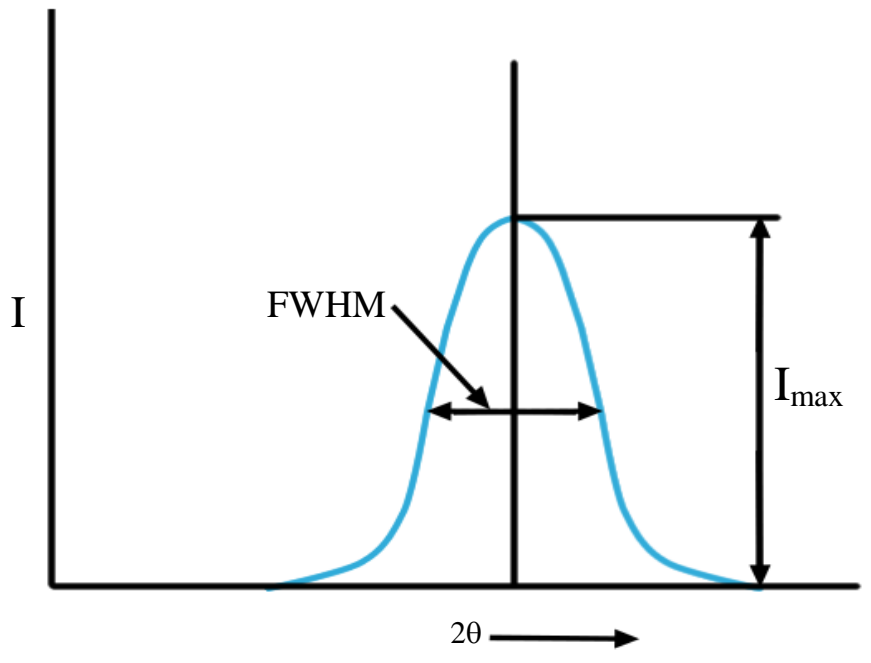

Fig. 8: At Bragg angle $\theta$, the diffraction peak is observed

The interaction between the atomic planes of the crystal and the X-rays are the synonymous with a uniform ruled grating and a beam of light (X-ray diffraction, 2013). This is also known as elastic or coherent scattering (He, 2018). Out of all the many theories and equations there are to describe the relationship between the diffraction pattern and the original substance structure, the simplest, in this case, is Bragg's Law. Bragg's Law states that $\mathrm{n} \lambda=2 \mathrm{~d} \sin \theta$, where $\mathrm{n}$ is an integer called the order of reflection, $\lambda$ is the wavelength, $\mathrm{d}$ is the distance between adjacent planes and $\theta$ is the Bragg angles where the diffraction peak is observed (Fig. 8) (He, 2018). The diffraction peak is observed when the incident X-rays strike the crystal plane at angle $\theta$ and reflect likewise at angle $\theta$.

\section{Transmission Electron Microscopy}

There are many different types of microscopes. Among these, the electron microscope is a type that uses radiation to resolve extremely small structures that would not otherwise be seen with just visible light (Alberts et al., 2017). One type of electron microscope is the Transmission Electron Microscope (TEM). This microscope has three main components: an electron gun, the image-generating system and the image recording system (Fig. 9) (Transmission Electron Microscop, 2018). The electron gun generates the electron beam that is focused onto the sample via the condenser. The image-generating system includes: the objective lens, movable stage and intermediate and projector lenses, which focus the electrons passing through the sample. This is what helps to create the real and highly magnified image. The image-recording system uses a fluorescent screen to convert the electron image into a form that is perceivable to the human eye. The fluorescent screen views and further focuses the image and a digital camera, also a part of the image-recording system, archives the image for permanent record (Transmission Electron Microscop, 2018).

Figure 10 shows the fluence dependence of Normal $\theta-2 \theta$ X-ray Diffraction patterns of the multilayer thin films. As seen from Fig. 10, all of the thin films, unannealed and annealed at different fluences, have similar peaks for their elemental contribution.

Figure 11 shows FIB milled SEM cross-section image when geometric transformations is off by UA support on bombarded multilayered thin film at the fluence of $1 \times 10^{13}$ ions $/ \mathrm{cm}^{2}$. Figure 12 shows FIB milled SEM cross-section image when geometric transformations is off by UA support on bombarded multilayered thin film at the fluence of $5 \times 10^{13}$ ions $/ \mathrm{cm}^{2}$.

Figure 13 and 14 show a large scale, starting perspective of all the layers together along with the diagram of the multilayer structure. This is to provide a perspective of all the structures that can be seen in the Transmission Electron Microscopy. In these, the consistency and density of the layers and the "dark spots" can be seen.

From these images, it should be noted that (1) the disordered layers at the top was a Pt layer added by UA during sample prep and (2) the images were NOT in the same scale (magnification) so the thicknesses should not be compared directly.

Figure 15 and 16 were closer images of what was believed to be the "Multilayer $\mathrm{Ge} / \mathrm{Si}+\mathrm{Ge}$ " structure. The bombarded at $1 \mathrm{E} 14$ ions $/ \mathrm{cm}^{2}$ appeared to be $\sim 250 \mathrm{~nm}$ thick while the unbombarded appeared to be $\sim 200 \mathrm{~nm}$ thick. The individual $\mathrm{Ge} / \mathrm{Ge}+\mathrm{Si}$ layers were not able to be differentiated.

In STEM higher atomic number elements show up brighter than relatively lower atomic number elements, so the Ge should show up brighter than the Si. Thus, "darker spots" in the multilayer were believed to be Si. These dark spots do not appear larger or more dense in the in either coating. 


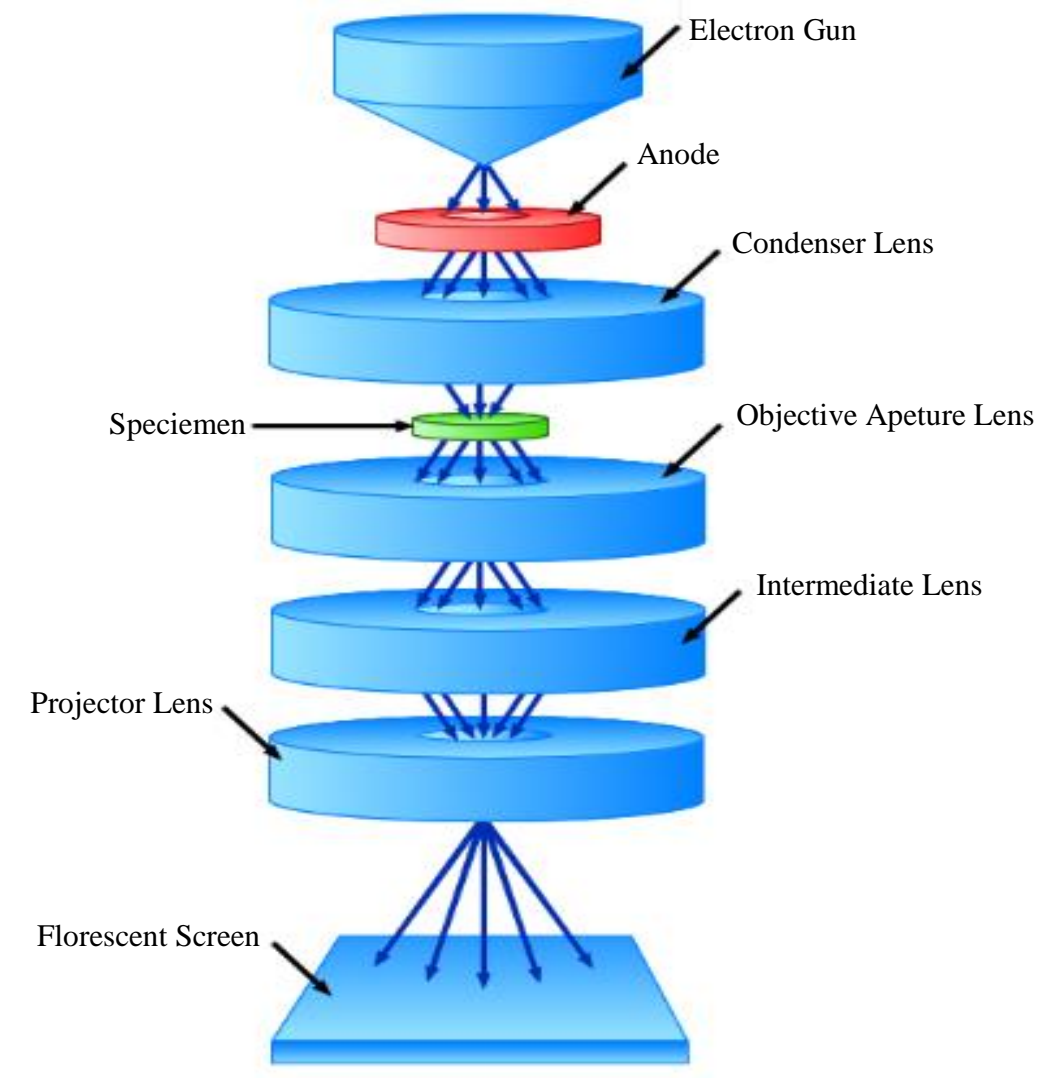

Fig. 9: Visual representation of a transmission electron microscope and its components

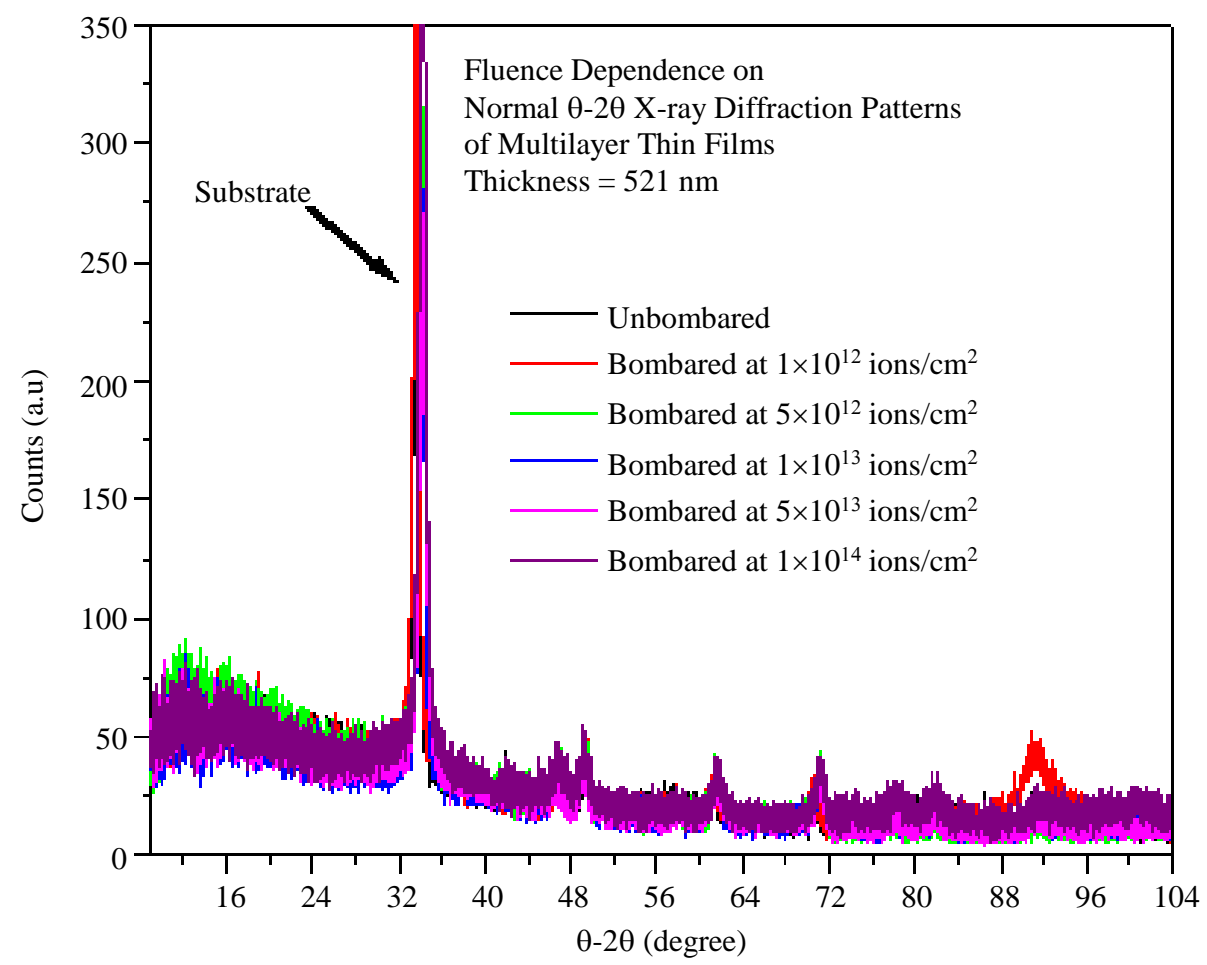

Fig. 10: Fluence dependence of normal $\theta-2 \theta \mathrm{X}$-ray diffraction patterns of the multilayer thin films 


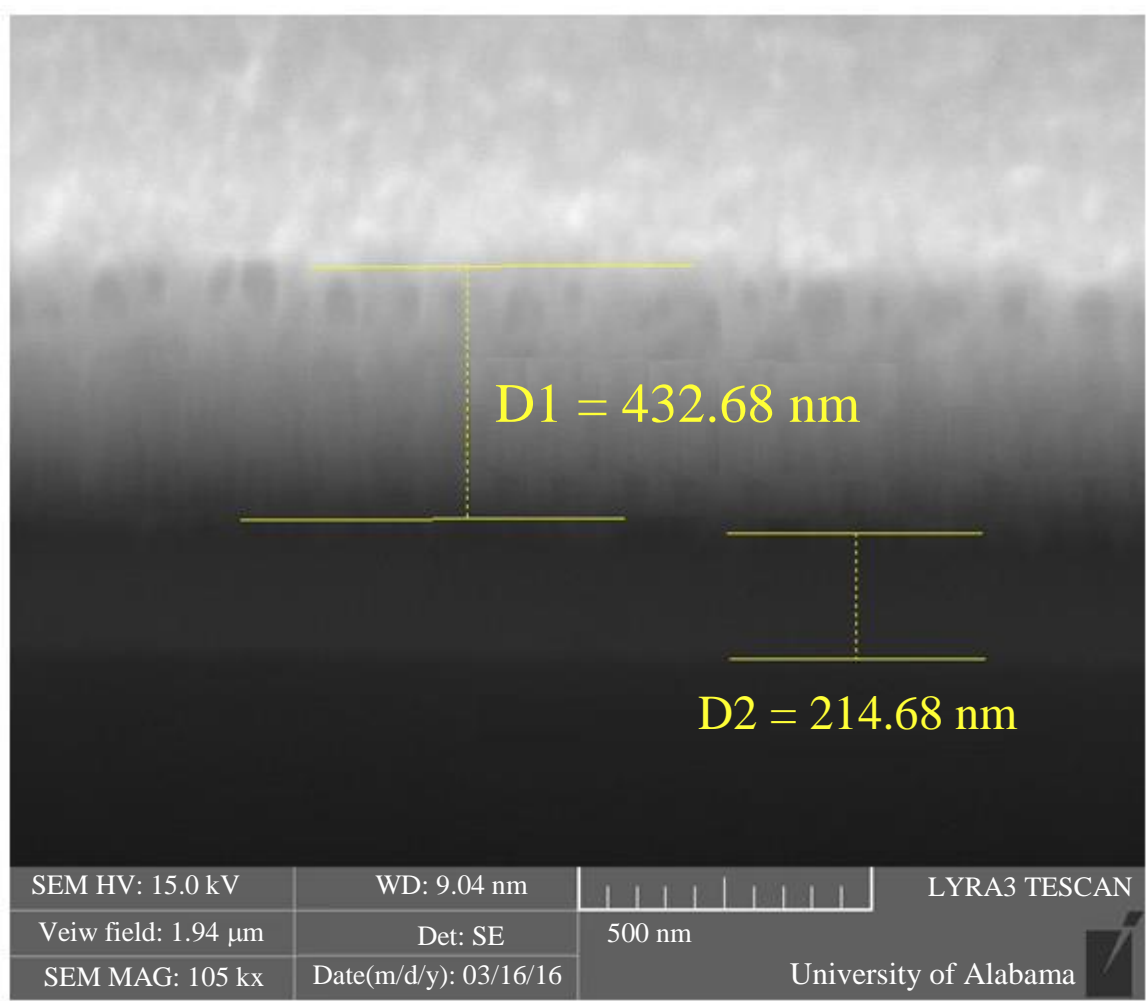

Fig. 11: FIB milled SEM cross-section image when geometric transformations is off by UA support on bombarded multilayered thin film at the fluence of $1 \times 10^{13}$ ions $/ \mathrm{cm}^{2}$

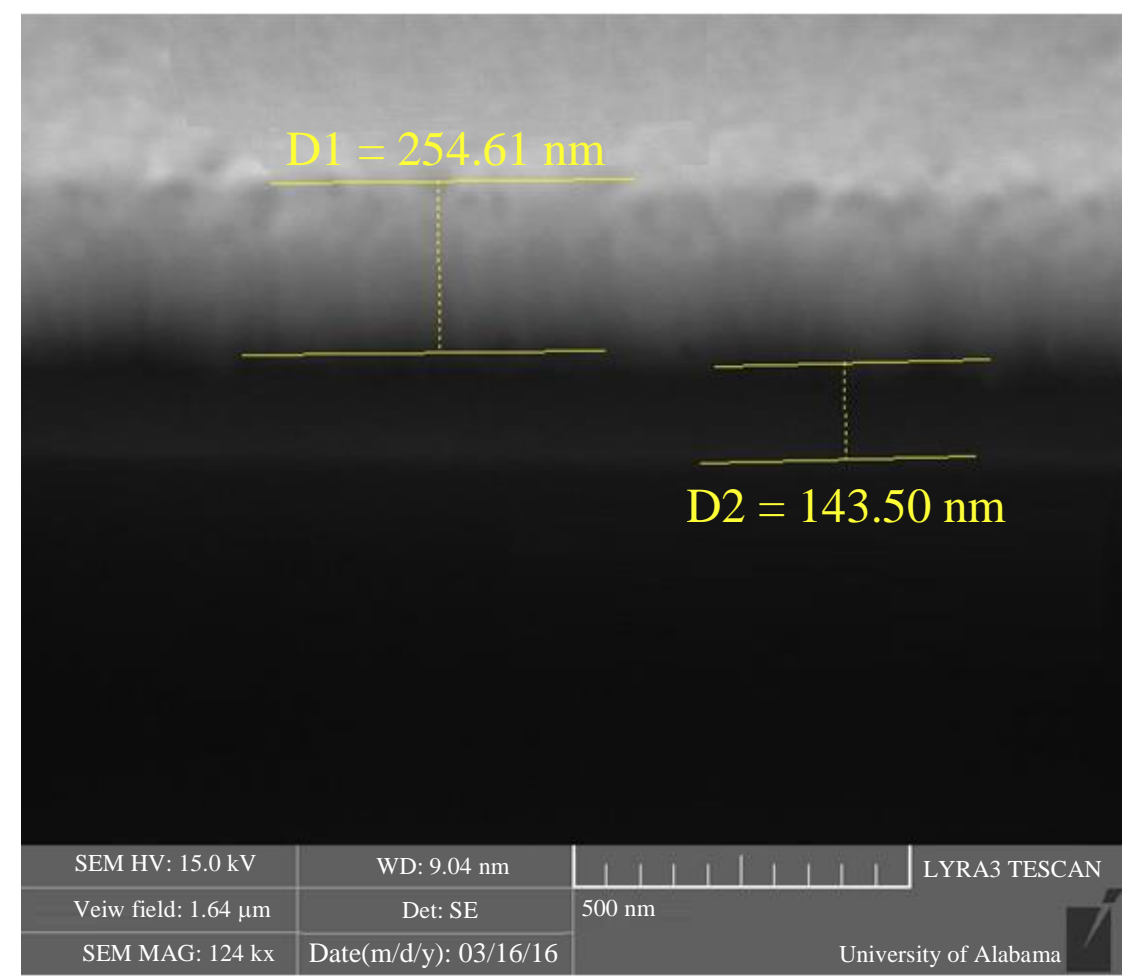

Fig. 12: FIB milled SEM cross-section image when geometric transformations is off by UA support on bombarded multilayered thin film at the fluence of $5 \times 10^{13}$ ions $/ \mathrm{cm}^{2}$ 
Mebougna Drabo and Satilmis Budak / American Journal of Applied Sciences 2019, 16 (8): 225.237 DOI: 10.3844/ajassp.2019.225.237

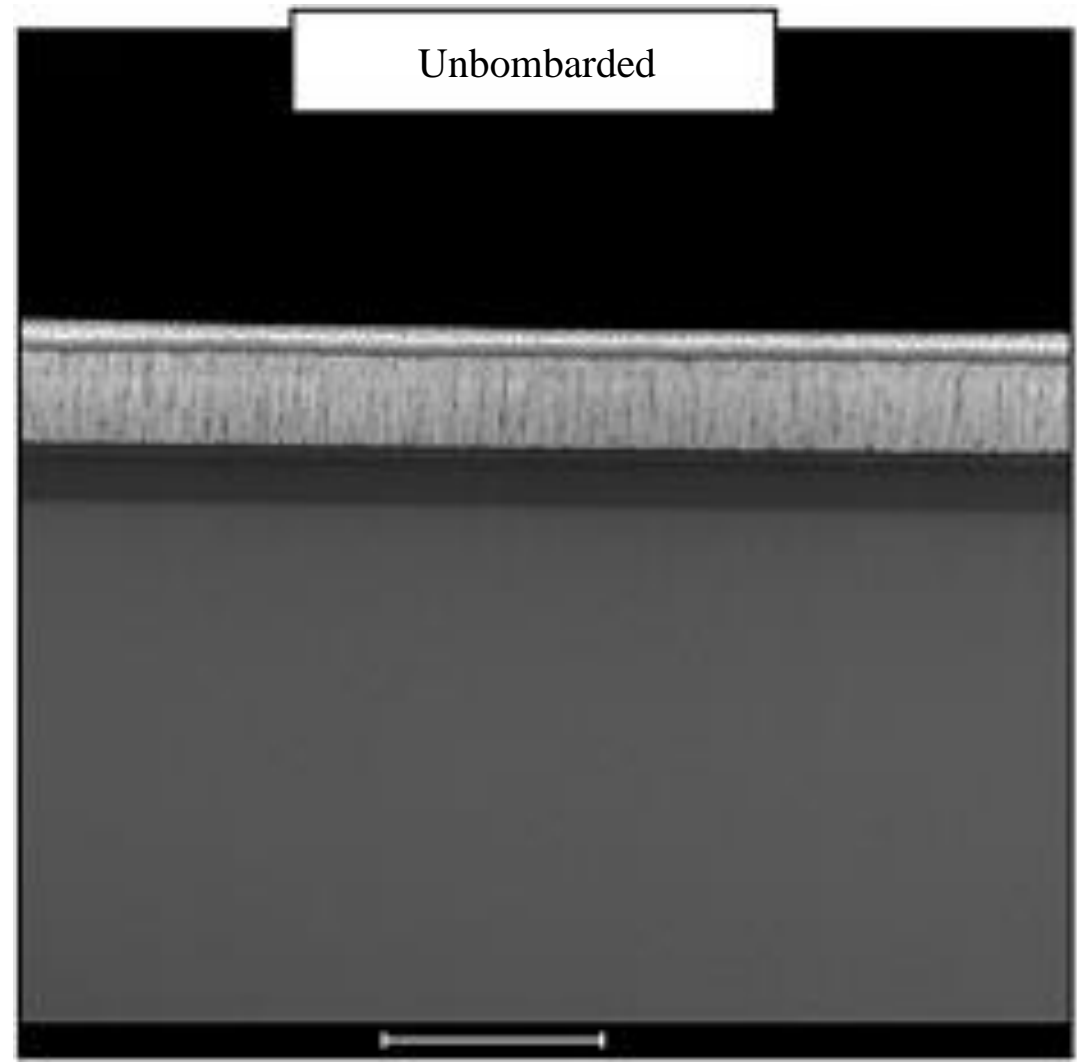

Fig. 13: Scanning Transmission Electron Microscopy for the multilayered thin film bombarded at the fluence of at $1 \times 10^{14}$ ions $/ \mathrm{cm}^{2}$

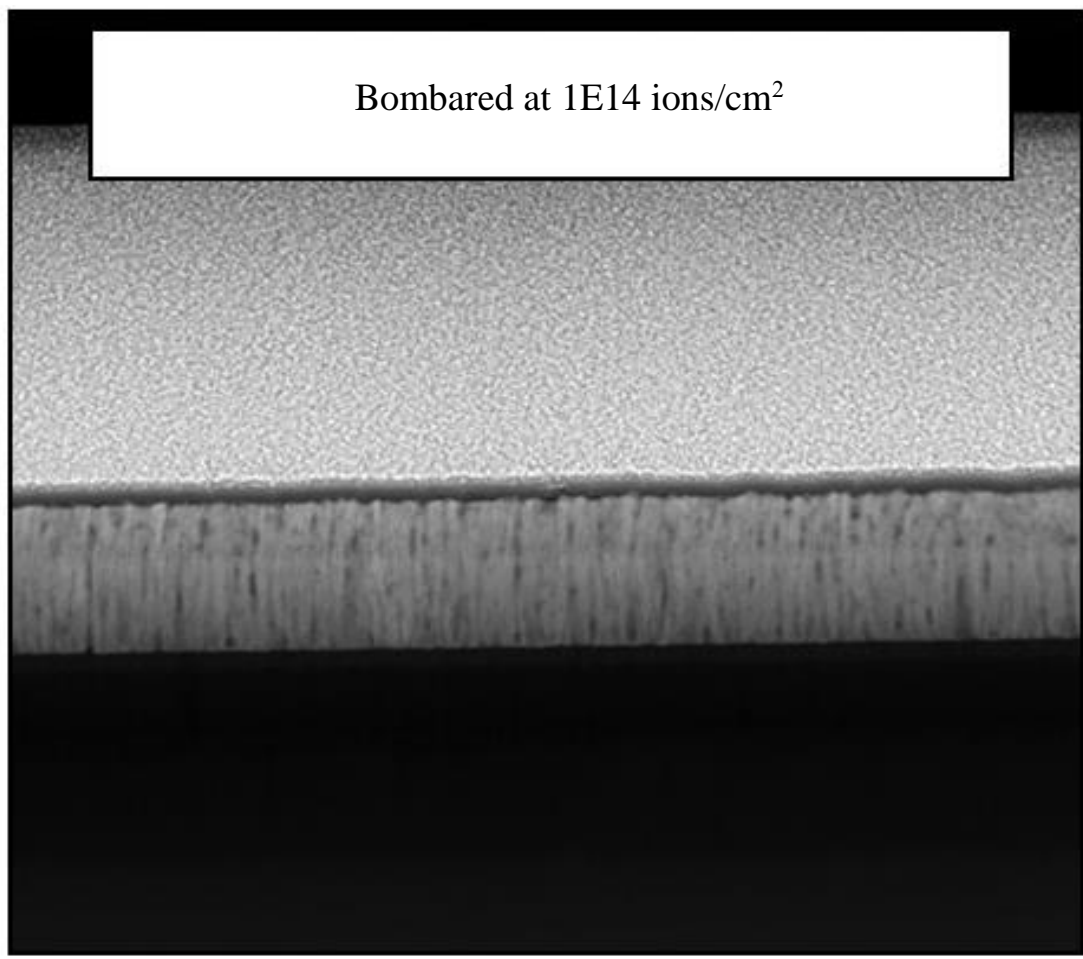

Fig. 14: Scanning Transmission Electron Microscopy for the unbombarded multilayered thin film 


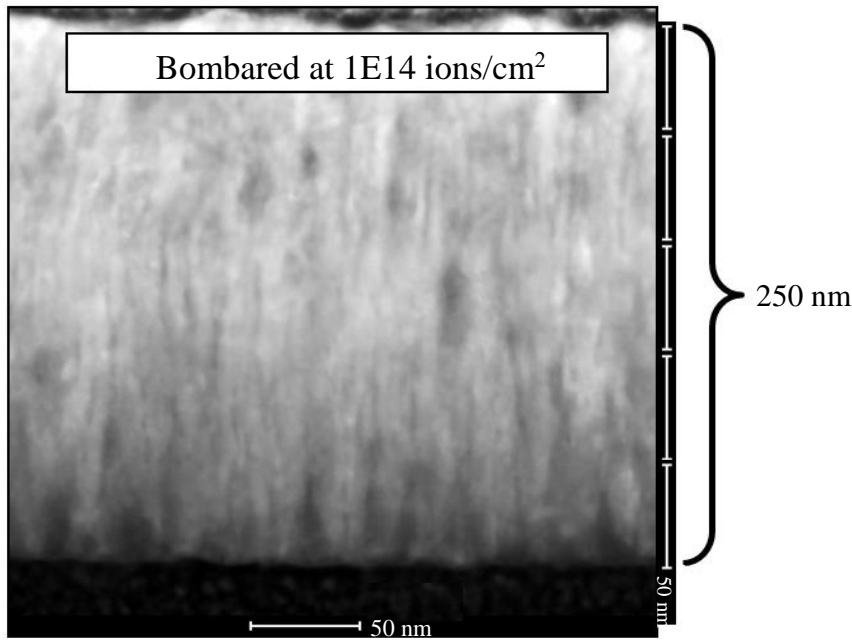

Fig. 15: Scanning Transmission Electron Microscopy for the multilayered thin film bombarded at the fluence of $1 \times 10^{14}$ ions $/ \mathrm{cm}^{2}$ (Thickness $=250 \mathrm{~nm})$

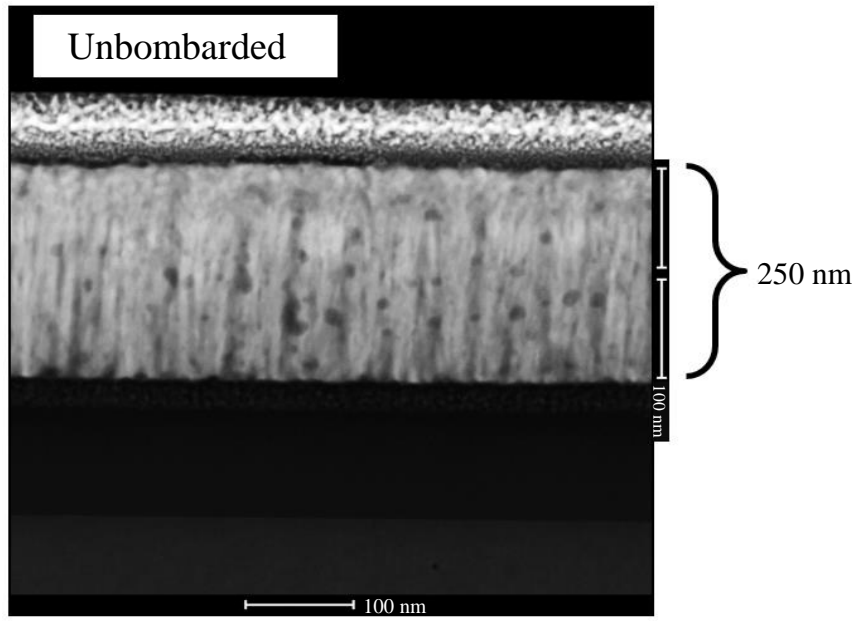

Fig. 16: Scanning Transmission Electron Microscopy for the unbombarded multilayered thin film (Thickness $=200 \mathrm{~nm}$ )

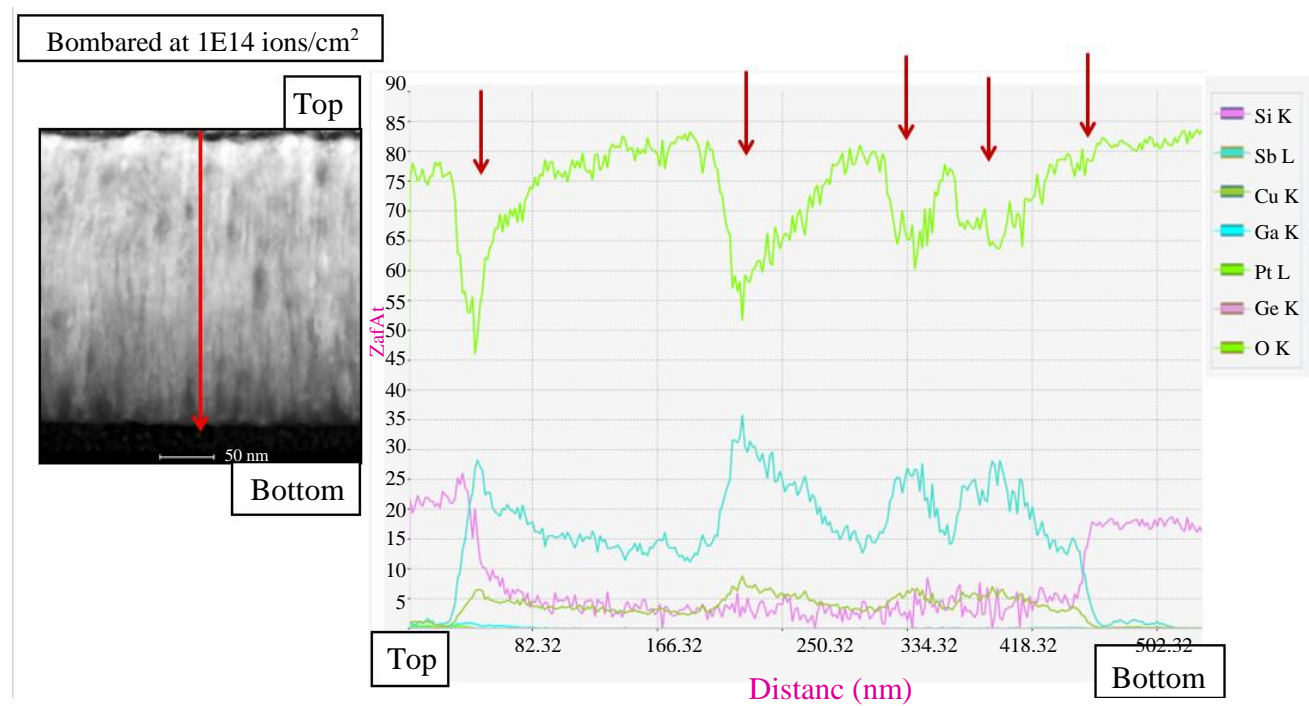

Fig. 17: Energy Dispersive X-Ray Spectroscopy for the multilayered thin film bombarded at the fluence of $1 \times 10^{14}$ ions $/ \mathrm{cm}^{2}$ 

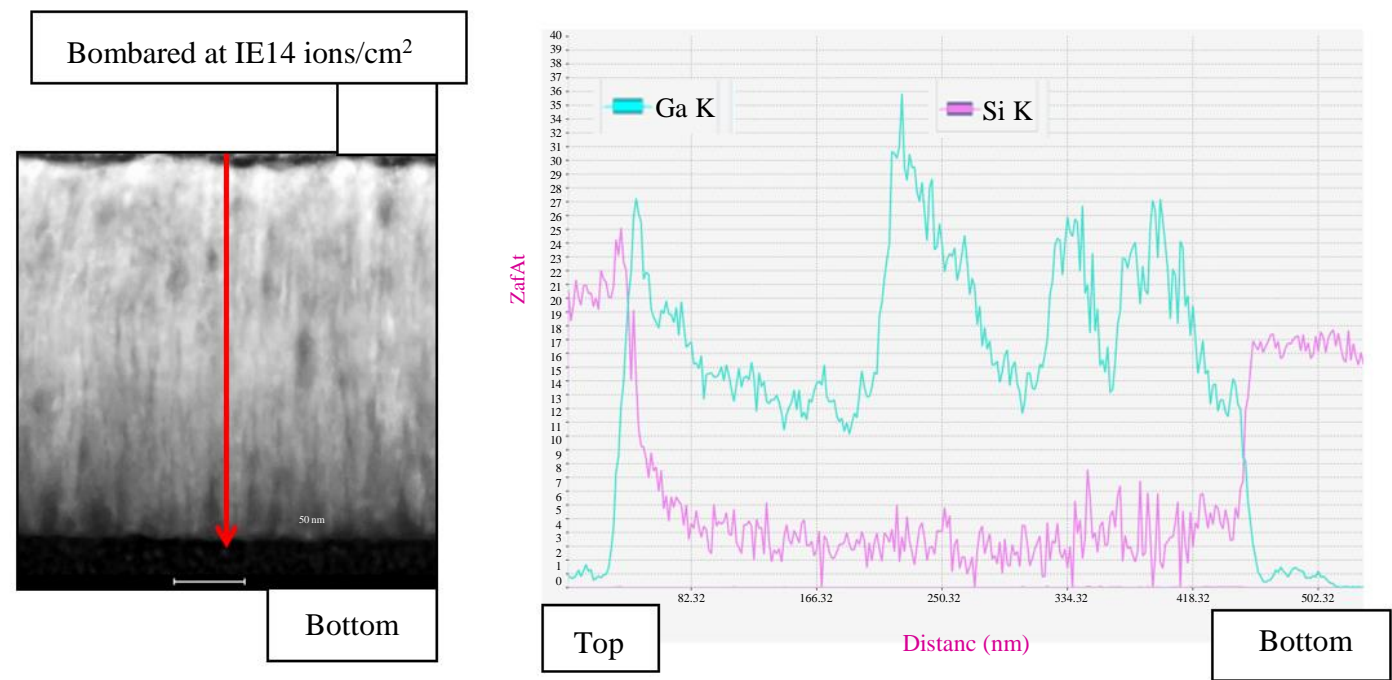

Fig. 18: Energy Dispersive X-Ray Spectroscopy for the multilayered thin film bombarded at the fluence of $1 \times 10^{14}$ ions $/ \mathrm{cm}^{2}$ for $\mathrm{Si} \mathrm{K}$ and $\mathrm{Ga} \mathrm{K}$

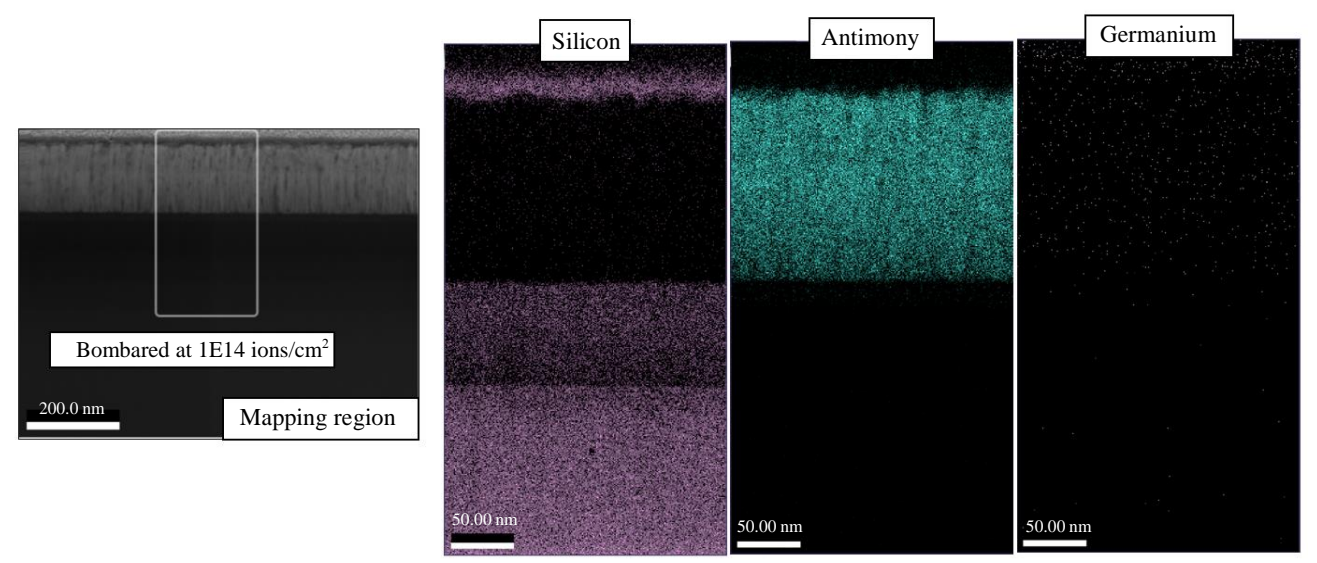

Fig. 19: EDS mapping for silicon, antimony and germanium

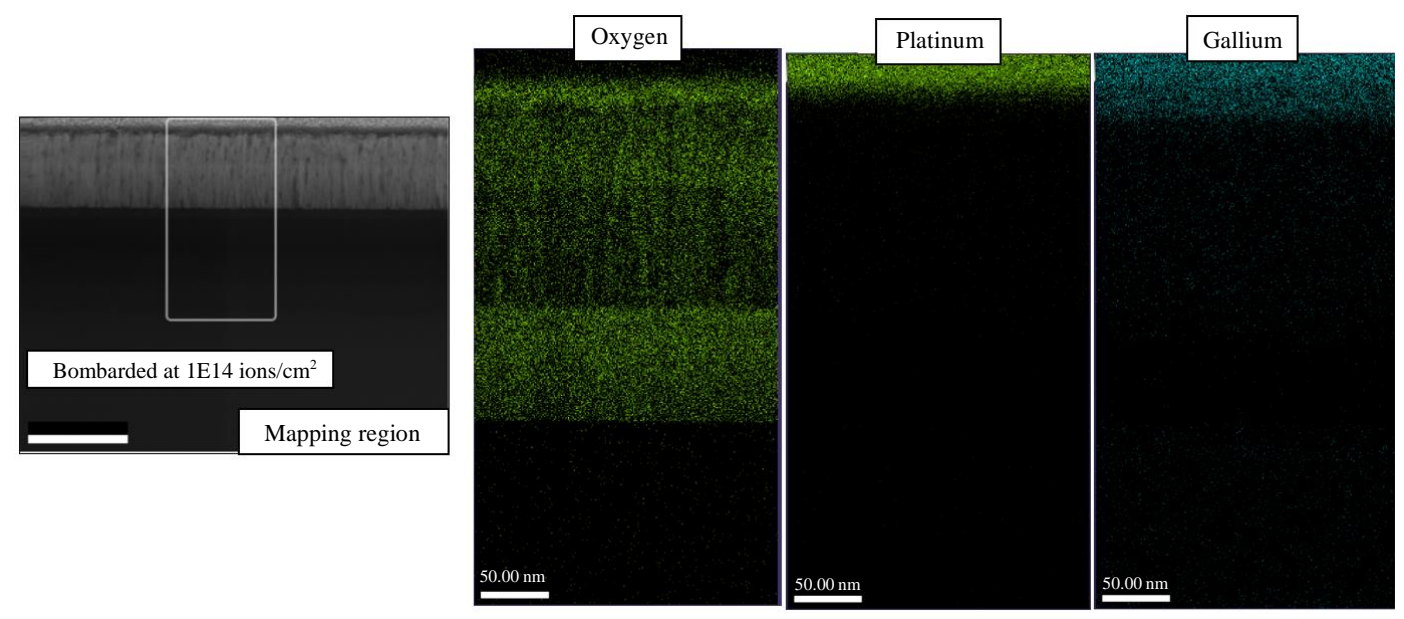

Fig. 20: EDS mapping for oxygen, platinum and gallium 


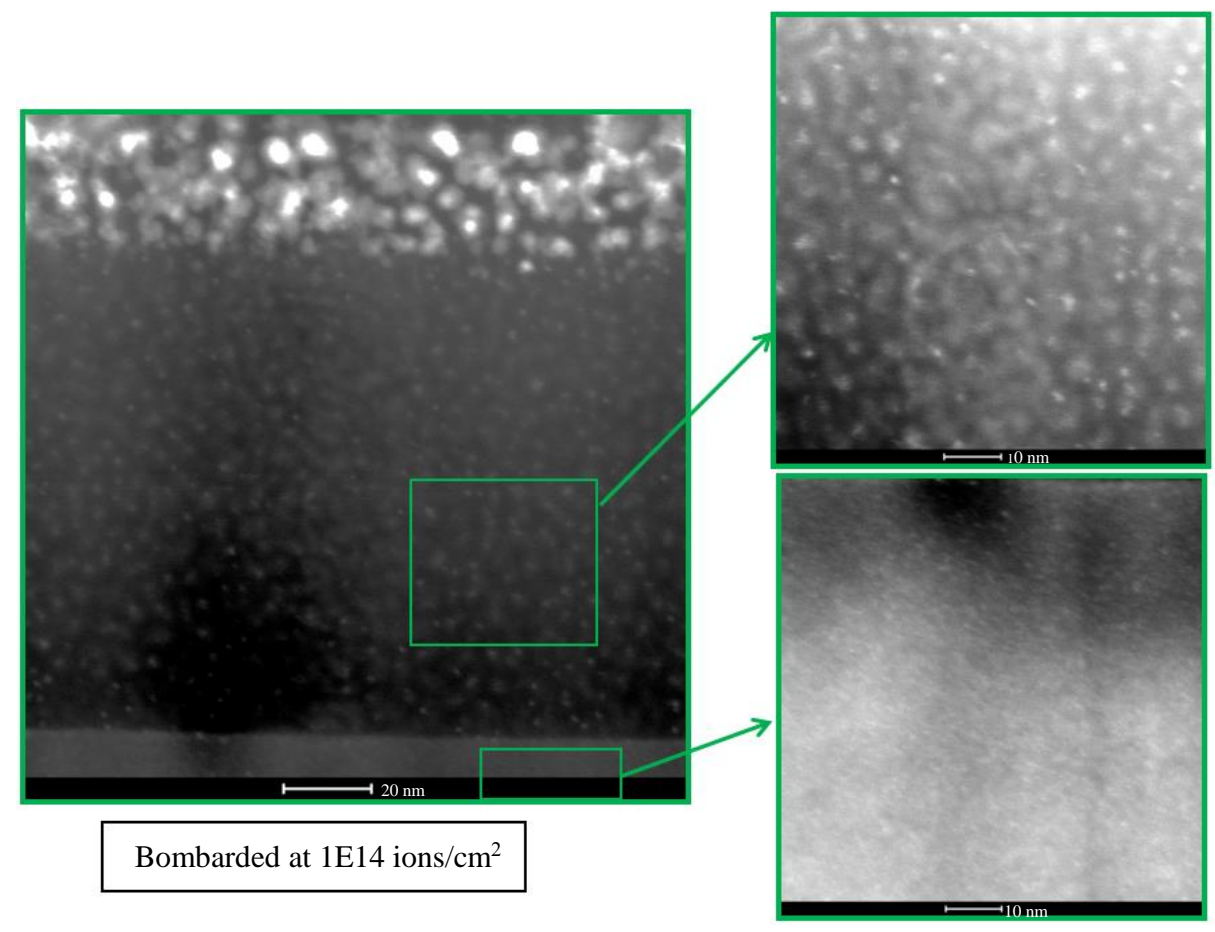

Fig. 21: Formation of Quantum Dots in the multilayer thin films during the high energy ion beam exposure

Figure 17 shows the Energy Dispersive X-Ray Spectroscopy for the multilayered thin film bombarded at the fluence of $1 \times 10^{14}$ ions $/ \mathrm{cm}^{2}$. Using EDS we were able to analyze the chemical makeup of the structures. In Fig. 17 we have taken a line scan (red arrow) from the top of the coating to the bottom, collecting many individual point scans along the line to show any changes or gradients in composition. The crimson arrows indicated possible locations of layer change, simply based on composition. The most important note is that oxygen, silicon and antimony were by far the most prevalent elements in found in this region.

Figure 18 shows the same EDS line scan data, but with only $\mathrm{Ga}$ and $\mathrm{Si}$ elements found in the multilayer diagram. From the first graph it was clearly seen the levels of $\mathrm{Ga}$ are incredibly low overall, while the $\mathrm{Si}$ levels were much lower only in the multilayer region.

From the second, the multilayer composition appears dominated by the $\mathrm{Sb}$ concentration. Note: $\mathrm{Y}$-axis is atomic concentration in percent and the $\mathrm{X}$-axis is distance from the top of the structure.

Figure 19 provides a wider field of view of the chemical species. The results from the mapping correlated well with the line scans from the previous slides. Figure 20 provides a wider field of view of the chemical species. The results from the mapping correlated well with the line scans from the previous slides. Figure 21, the structures are from the two layers directly below the multilayer structure and are the only structures that showed and "dot-like" structures.

\section{Conclusion}

The highest power factor could be reached when the high energy beam dose (fluence) was $1 \times 10^{13}$ ions $/ \mathrm{cm}^{2}$. It seems that the fluence of $1 \times 10^{13}$ ions $/ \mathrm{cm}^{2}$ looks like a turning point since the power factor increases until this fluence and then the power factor started decrease after this fluence. The similar behaviors could be seen for the electrical conductivity values. The highest thermal conductivity could be reached when the high energy beam dose (fluence) was $1 \times 10^{13}$ ions $/ \mathrm{cm}^{2}$. It seems that the fluence of $1 \times 10^{13}$ ions $/ \mathrm{cm}^{2}$ looks like a turning point since the thermal conductivity increases until this fluence and then the thermal conductivity started decrease after this fluence. Efficient thermoelectric materials and devices could have high Seebeck coefficient and electrical conductivity and lower thermal conductivity values. After the fluence of $1 \times 10^{13}$ ions $/ \mathrm{cm}^{2}$, the thermal conductivity values decrease. This a good sign for the efficient thermoelectric devices. The highest figure merit was calculated when the fluence was $1 \times 10^{13}$ ions $/ \mathrm{cm}^{2}$ like the highest power factor and highest thermal conductivity were measured at this fluence. Even if we reached the highest thermal conductivity at this fluence, we reached also the highest figure of merit due to the highest values of power factor at this fluence. More studies have been performed on the film systems including Normal $\theta-2 \theta$ X-ray Diffraction patterns, FIB milled SEM cross-section images and Scanning Transmission Electron Microscopy (STEM). Ion beam 
modification cause nano-dots like formation in the multilayer thin films systems and they have been shown in STEM images. Formation of nanostructures in the multilayer structures could cause increase in both Seebeck coefficient and electrical conductivity and decrease in the thermal conductivity at the suitable fluence of ion beam bombardment

\section{Acknowledgement}

The author would like to thank the Central Analytical Facility at the University of Alabama, Tuscaloosa, AL USA for their support and we thank also our undergraduate students, Ashley Tramble and Chauncy Casselberry for their attendance during the data gathering.

\section{Author's Contributions}

Each author of this manuscript made considerable contributions in conducting the experimental testing, dataanalysis and contributed to the writing of this manuscript.

\section{Funding Information}

Research was sponsored by NSF with grant numbers NSF-HBCU-RISE-1546965, NSF-EPSCOR-R-II-3-EPS1158862, DOD with grant numbers W911 NF-08-1-0425 and W911NF-12-1-0063, U.S. Department of Energy National Nuclear Security Administration (DOE-NNSA) with grant numbers DE-NA0001896 and DE-NA0002687.

\section{Ethics}

All rights reserved. No part of this publication may be reproduced or transmitted in any form or by any means, electronic or mechanical, including photocopy, or any information storage and retrieval system, without permission in writing from the publisher or authors.

\section{References}

Alberts, B., D. Bray, K. Hopkin, A.D. Johnson and J. Lewis et al., 2017. Molecular biology of the cell. Garland Sci.
Budak, S., E. Gulduren, B. Allen, J. Cole and J. Lassiter et al., 2015. High energy radiation effects on the seebeck coefficient, van der pauw-hall effect parameters and optical properties of $\mathrm{Si} / \mathrm{Si}+\mathrm{Sb}$ multinanolayered thin films. Am. J. Mater. Sci., 5: 39-47.

Budak, S., K. Heidary, R.B. Johnson, T. Colon and C. Muntele et al., 2014. MeV Si ion modifications on the thermoelectric generator from $\mathrm{Si} / \mathrm{Si}+\mathrm{Ge}$ nanolayered films. Applied Surface Sci., 310: 221-225.

Budak, S., R. Parker, C. Smith, C. Muntele and K. Heidary et al., 2013. Superlattice multi-nanolayered thin films of $\mathrm{SiO}_{2} / \mathrm{SiO}_{2}+\mathrm{Ge}$ for thermoelectric device applications. J. Intel. Mater. Syst. Structures, 24: 1357-1364.

Budak, S., Z. Xiao, B. Johnson, J. Cole and M. Drabo, 2016. Highly-efficient advanced thermoelectric devices from different multilayer thin films. Am. J. Eng. Applied Sci., 9: 356-363.

DOI: 10.3844/ajeassp.2016

Chen, G., M.S. Dresselhaus, G. Dresselhaus, J.P. Fleurial and T. Caillat, 2003. Recent developments in thermoelectric materials. Int. Mater. Rev., 48: 45-66.

He, B.B., 2018. Two-Dimensional X-ray Diffraction. 1st Edn., John Wiley and Sons.

Pichanusakorn, P. and P. Bandaru, 2010. Nanostructured thermoelectrics. Mater. Sci. Eng., 67: 19-63.

Transmission Electron Microscop, 2018. Retrieved from encyclopedia britannica online database. Transmission Electron Microscope.

X-ray Diffraction, 2013. Retrieved from encyclopedia britannica online database.

Zhang, X. and L.D. Zhao, 2015. Thermoelectric materials: Energy conversion between heat and electricity. J. Materiom., 1: 92-105. 